\title{
Low Power and Power Aware Fractional Motion Estimation of H.264/AVC for Mobile Applications
}

\author{
Tung-Chien Chen, Yu-Han Chen, Chuan-Yung Tsai and Liang-Gee Chen \\ DSP/IC Design Lab., Graduate Institute of Electronics Engineering and Department of Electrical \\ Engineering, National Taiwan University; Email: djchen, doliamo, cytsai, 1gchen@ video.ee.ntu.edu.tw
}

\begin{abstract}
In this paper, the low power design techniques from algorithm to architecture levels are proposed for factional motion estimation in H.264/AVC. The proposed AMPD algorithm can reduce 50.8\% power with up to $0.1 \mathrm{~dB}$ quality drop. The proposed parallel architecture with efficient memory hierarchy can efficiently reuse data and save $61.6 \%$ power. Furthermore, the power aware functionality is included. Our design can gracefully vary the quality degradation of $0.1-3.9 \mathrm{~dB}$ in response to the 22.58-1.64 $\mathrm{mW}$ power consumption. This power-oriented design is very efficient for different mobile applications in various power situations.
\end{abstract}

\section{INTRODUCTION}

While highly interactive and recreational multimedia applications appear much faster than expected, there seems to be an inexhaustible demand for as much higher compression ratio and better video quality as possible. The new video coding standard of H.264/AVC [1], which significantly outperforms previous standards, undoubtedly plays an important role in this area. The new techniques [2] of 1/4-pixel resolution, variable block sizes (VBS), multiple reference frames (MRF), and Lagrangian mode decision in motion estimation (ME) induce huge computation complexity, and hardware acceleration is a must.

Classical hardware architecture focuses on latency, area, throughput and hardware complexity as the primary parameters to be optimized or traded off against each other. However, power-oriented design methodology has emerged as a key factor for two reasons: limited availability of power in portable or wearable devices, and limited capacity to dispose of the heat of VLSI circuits. Low power and power aware are the most important issues in the power-oriented design. The low-power ME can provide the maximum compression performance under the specific power constraint. The power-aware ME can extent the battery life by varying the compression performance and power consumption according to the system power status. Many power-oriented fast algorithms and hardware architecture have been proposed for integer motion estimation (IME) [3] [4] [5], but none for fractional motion estimation (FME). However, according to our analysis, the FME occupies $45 \%$ [6] of the runtime in H.264/AVC inter prediction and upgrades rate-distortion efficiency by $2-6 \mathrm{~dB}$ in peak signal to noise ratio (PSNR). The advanced FME design with optimization in power issues is urgently demanded by H.264/AVC compression system.

In this paper, the first low-power and power-aware FME design of H.264/AVC is proposed. The rest of this paper is organized as follows. In Section II, the related power reduction and power aware techniques will be described. In Section III, the power-aware fast algorithm are proposed. The corresponding parallel hardware architecture is designed in Section IV with low-power considerations, while the implementation and simulation results are presented in Section V. Finally, Section VI

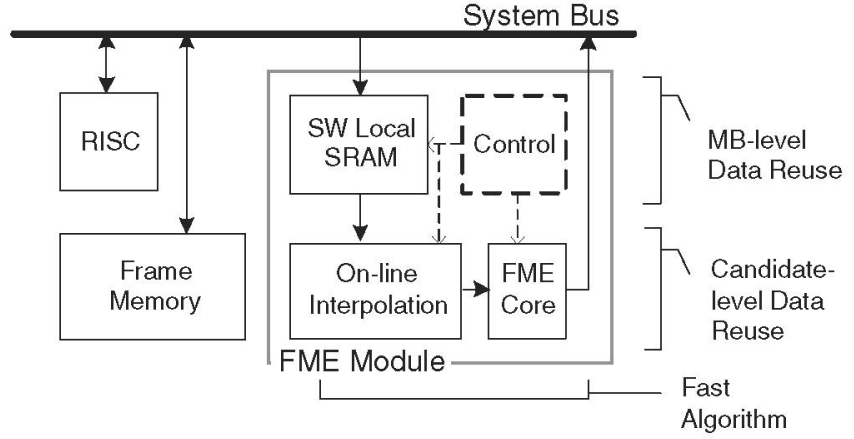

Fig. 1. FME system architecture and the corresponding power reduction techniques.

gives a conclusion.

\section{FUNDAMENTAL}

\section{A. FME System Architecture and Power Reduction Techniques}

Figure 1 shows the FME system architecture. In reference software [7], in order to support ME with quarter-pel resolution, all required pixels are interpolated in advance and stored in the frame memory. However, the storage space with 16-times of frame size is required for each reference frame, which is a considerable overhead for hardware implementation. Besides, the system bandwidth (BW) will become too large during FME procedure. Therefore, the on-chip on-line interpolation is generally adopted [6]. In this system structure, the power consumption mainly comes from two parts. One is data access power of integer reference pixels loading from frame memory to FME core. The other is computation power of interpolation and matching cost calculation.

Several power reduction techniques are described as follows. First, because the referred search areas of neighboring current MBs (CMBs) are considerably overlapped, the search window (SW) SRAMs are firstly embedded to achieve MB-level data reuse (DR). The BW and power of system memory can be reduced. Second, the parallel architecture is designed to achieve candidate-level DR, and the BW and power of local memory can be reduced. Third, the hardware-oriented fast algorithm can be used to reduce the computational power of interpolation engine and FME core. Last but not least, the gated clock technique can be applied to turn the inoperative circuits off.

\section{B. Power Aware Functionality}

Power aware functionality is another important issue for power-oriented design. It can vary the power consumption according to the power status at the expense of compression per- 


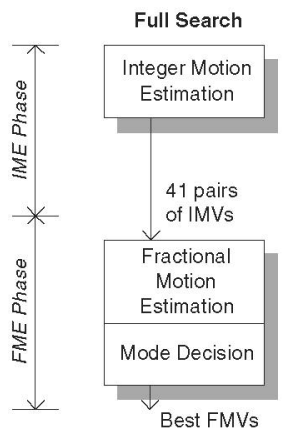

(a)

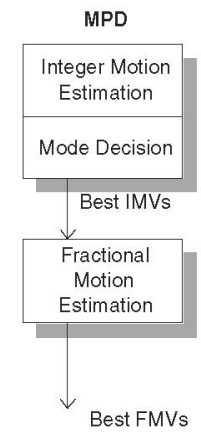

(b)

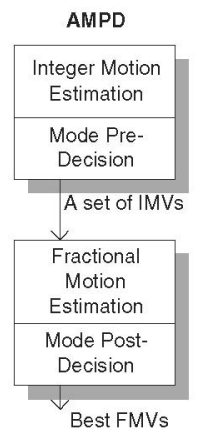

(c)
Fig. 2. (a) FS algorithm; (b) MPD fast algorithm; (b) Proposed AMPD fast algorithm.

formance. For H.264/AVC standard, power aware functionality becomes more necessary. That is because the computation complexity of the video compression algorithm grows much faster than the progress of power supply. A specified power constraint are usually met at the expense of considerable quality drop, but high quality is usually claimed especially when power is full. The encoder with power aware functionality can not only have more adaptability for good balance between compression performance and power consumption but also extend the battery life time. In a well-designed power aware system, the compression performance should be varied gracefully in response to the power status. In order to maximize the compression performance under the power constraint, the processing tasks should be turned off according to the priority.

\section{Power AWARE FAst Algorithm}

Full search (FS) algorithm can guarantee the highest compressing performance. However, the large power consumption is very critical for many portable applications. In this section, a hardware oriented fast algorithm will be proposed with the power aware functionality.

Figure 2 (a) shows the concept of FS algorithm. H.264/AVC supports VBS, and the inter mode decision with FS algorithm is done with the costs of all blocks and sub-blocks, which are refined toward quarter-pel resolution. In order to reduce the computation complexity, a simple fast algorithm denoted as Mode Pre-Decision (MPD) was evaluated firstly. As shown in Fig. 2 (b), the inter mode decision is moved forward to IME phase. That means the selection of the best combination of VBS is done in integer-pel resolution. The FME is thus responsible for refining the integer motion vectors (IMVs) of the selected mode toward quarter-pel resolution. In VBS, each MB can be split up into 4 kinds of partition: $16 \times 16,16 \times 8,8 \times 16$, and $8 \times 8$. If partition $8 \times 8$ is selected, each block can be further split into 4 kinds of sub-partitions: $8 \times 8,8 \times 4,4 \times 8,4 \times 4$. Totally 41 blocks and sub-blocks are involved per reference frame and have totally seven-times pixels of one MB. Therefore, the MPD can reduce about $6 / 7$ computation complexity. However, the rate-distortion efficiency is seriously degraded for up to one dB in PSNR. That is because the best combination of VBS in integer-pel resolution may change after the refinement in quarter-pel resolution.

To achieve a better trade-off between quality and computation complexity, advanced MPD (AMPD) algorithm is proposed. As shown in Fig. 2 (c), the inter mode decision is divided into

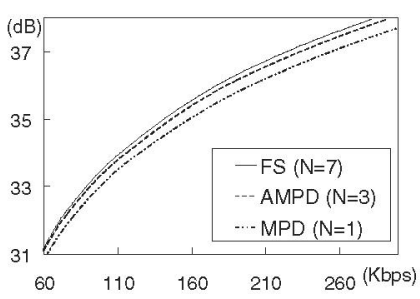

(a) Foreman (QCIF)

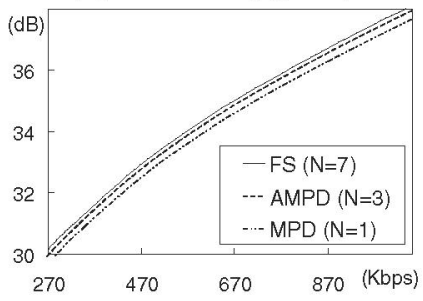

(a) Mobile (QCIF)

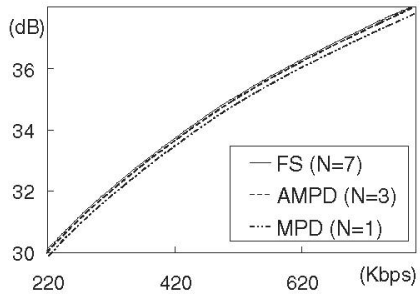

(b) Stefan (QCIF)

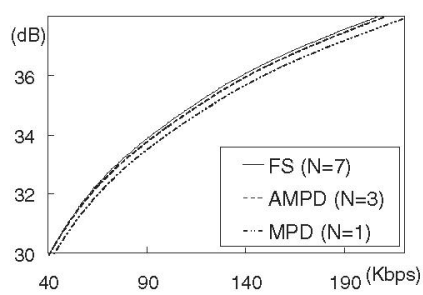

(b) Table (QCIF)
Fig. 3. Rate-distortion curves of FS, MPD, and AMPD algorithms. The parameters are QCIF, 30frames/s, 1 reference frames, \pm 16 -pel search range (SR), and low complexity mode decision.

"Mode Pre-Decision" and "Mode Post-Decision". In "Mode Pre-Decision", not only one but a set of probable modes are selected after IME phase. Firstly, the four sub-modes of each $8 \times 8$ block are sorted according to the Lagrangian matching costs in integer-pel resolution. The costs of the best sub-modes of four $8 \times 8$ blocks are then summed up as one $8 \times 8$ partition costs. The costs of the second sub-modes are summed up as the second $8 \times 8$ partition costs, and so on. There are totally four costs of the $8 \times 8$ partitions. Secondly, the costs of seven partition, four $8 \times 8$ partitions together with the $16 \times 8,8 \times 16$, and $16 \times 16$ partitions, are sorted from low (best) to high (worst). $N(N=1 \sim 7)$ of the best partitions are selected for the FME procedure followed by "Mode Post-Decision". Note that, if more than one $8 \times 8$ partitions are selected, the re-combination among the submodes is allowed in "Mode Post-Decision". When $\mathrm{N}$ is equal to one, AMPD has the same performance with MPD. When $\mathrm{N}$ is equal to seven, AMPD has the same performance with FS.

Figure 3 shows the rate-distortion curves of AMPD, in which $\mathrm{N}$ is set to three, together with FS and MPD. The rate-distortion efficiency improves with the increase of $\mathrm{N}$, and so does the computation complexity. However, this improvement saturates when $\mathrm{N}$ is set to three. That means when $\mathrm{N}$ is larger than three, much more computation complexity must be paid with just the limited quality improvement, which is not efficient for power-oriented design. Therefore, the parameter of " $N=3$ " is chosen. Similarly, as for MRF-ME, the fast algorithm modified from [8] is used. According to the analysis in [8], more than $80 \%$ of the best reference frames have the temporal distance that is smaller than two. When the maximum reference frame number is set over than two, the quality improvement is limited with considerably increased computation complexity. Therefore, the maximum number of MRF is set to two in our design.

The AMPD can efficiently support power aware functionality. Because the correlation between mode decision in integer-pel resolution and that in quarter-pel resolution is very high. The priority of the VBS can be precisely estimated by the sorting operation in the "Mode Pre-Decision". By varying the parameter $\mathrm{N}$, our algorithm can have a good trade-off between power 


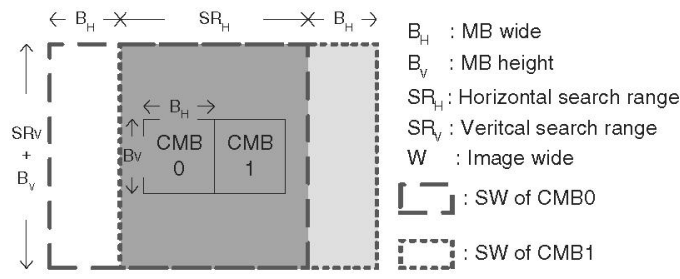

(a) Level-C DR Scheme

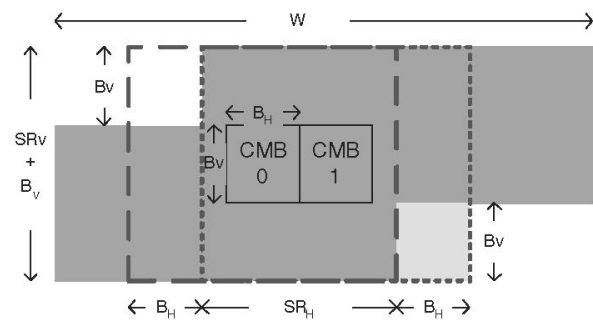

(b) Level-D DR Scheme

Fig. 4. MB-level DR schemes. When process is changed from CMB0 to CMB1, the dark gray region is the reused data, and the light gray region is the required data loaded from system memory.

consumption and compression performance. Furthermore, if the power is very low, we can even support only half-pel or integerpel resolution with one reference frame. In the later case, the FME module will only take care of the MC task. The quality approaches to MPEG-4 simple profile in this situation.

\section{Low Power Architecture Design}

In this section, the low-power design methodology on architecture level is described. The parallel architecture with efficient memory hierarchy is used. Two factors are used to evaluate the performance of the proposed low-power architecture: the redundancy access factors (RAs) for MB-level DR and candidatelevel DR, which are defined as follows.

$$
\begin{aligned}
R A_{\text {mb-level }} & =\frac{\text { System } B W \text { for loading } S W S}{\text { minimum requirement }} \\
R A_{\text {cand.-level }} & =\frac{S W \text { SRAM } B W \text { for reading ref-pels }}{\text { minimum requirement }}
\end{aligned}
$$

\section{A. MB-level Data Reuse}

For MB-level DR, four strategies have been proposed with different tradeoffs between local memory size and system bus BW and are indexed from level-A to level-D [9]. Because the power consumption to access the system memory is the most critical part, the level-C and level-D, that have lower RA factors, are recommended for memory technology nowadays. The level-C scheme reuses the horizontally overlapped region between two SWs of the neighboring CMBs as shown in Fig. 4 (a). Only the light gray part of $B_{H} \times\left(S R_{V}+B_{V}\right)$ pixels is required to loaded from external memory for every CMB. The Ra of Level-C scheme can be calculated as:

$$
R A_{m b-l e v e l, \text { level-C }} \approx \frac{B_{H} \times\left(S R_{V}+B_{V}\right)}{B_{H} \times B_{V}}=1+\frac{S R_{V}}{B_{V}}
$$

The required SW SRAMs' size is $\left(S R_{H}+B_{H}\right)\left(S R_{V}+B_{V}\right)$ pixels for each reference frame. As for the Level-D scheme, it can fully reuse the horizontally and vertically overlapped SWs of

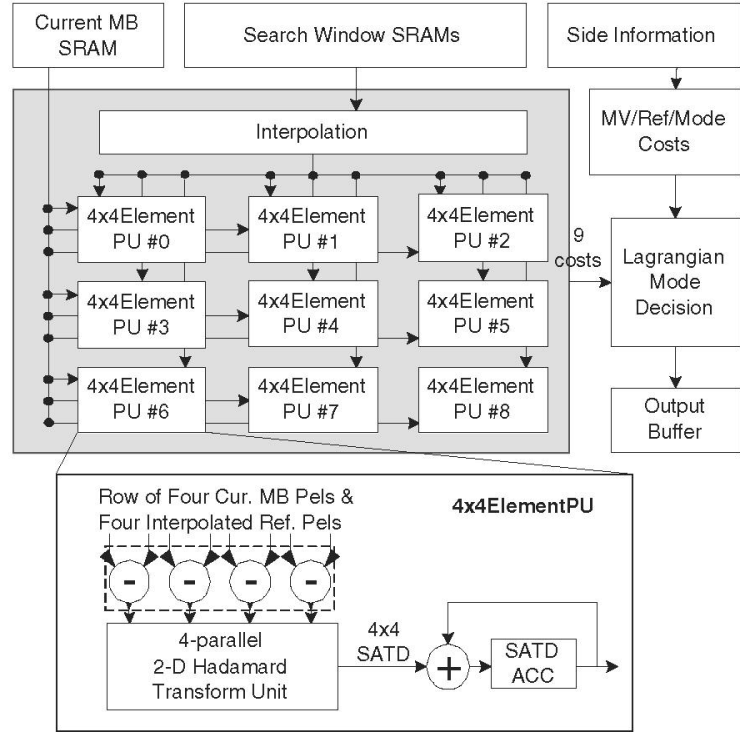

Fig. 5. The proposed parallel hardware with candidate-level DR. TABLE I

PERFormanCE OF CANDIDATE LEVEL DR FOR THE PROPOSED PARALLEL ARCHITECTURE.

\begin{tabular}{lcccc}
\hline Technology & $\begin{array}{c}\text { No } \\
\text { Paral. }\end{array}$ & $\begin{array}{c}\text { Intra-cand. } \\
\text { level DR }\end{array}$ & $\begin{array}{c}\text { Inter-cand. } \\
\text { level DR }\end{array}$ & Both \\
\hline RA $_{\text {candidate-level }}$ & 74.98 & 28.12 & 10.18 & 3.64 \\
\hline
\end{tabular}

neighboring CMBs. According to Fig. 4 (b), the RA of Level-D scheme is calculated as:

$$
R A_{m b-\text { level, level-D }} \approx \frac{B_{H} \times B_{V}}{B_{H} \times B_{V}}=1
$$

The Level-D scheme can minimize the system memory BW, but a huge local memory size of $\left(W \times S R_{V}\right)+\left(S R_{H}+B_{H}\right) \times B_{v}$ pixels is required for each reference frame.

In our design, both the level-C and level-D schemes are embedded and chosen according to the MRF number. In general case, two reference frames are used for the highest compression performance. Each reference frame is stored in SW SRAMs and reused in level-C scheme, separately. When the power is low, the SW SRAMs are configured as the level-D scheme for lowest power consumption in system bus. In this situation, only one reference frame can be supported.

\section{B. Candidate-level Data Reuse}

In H.264/AVC, FME supports quarter-pixel accuracy. After the integer ME, the half-pixel MV refinements are performed around the best integer search positions with $\pm 1 / 2$ pixel SR. The quarter-pixel ME, as well, is then performed around the best half search position with $\pm 1 / 4$ pixel SR. Therefore, each half and quarter refinement has nine candidates including the refinement center and its eight neighborhoods. For AMPD algorithm, this refinement procedure will be iteratively processed on the selected blocks and sub-blocks. The best mode is selected by considering both the distortion of SATD and the rate parts.

Based on the analysis in [6], the hardware architecture is shown in Fig. 5. The $4 \times 4$ block is the smallest element of VBS, and the SATD is also based on $4 \times 4$ blocks. Every block and subblock can be decomposed into several $4 \times 4$-elements with 


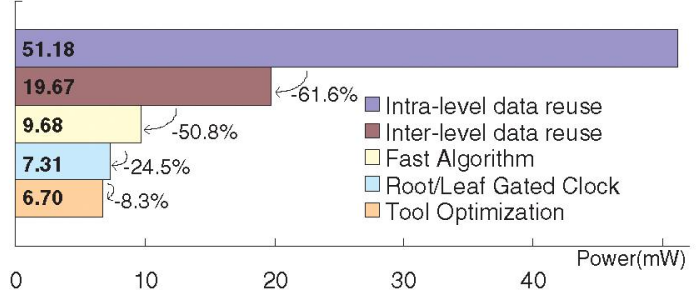

Fig. 6. Performance of the power reduction techniques on each level. The parameters are foreman $\mathrm{CIF}$ video, 30frames/s, 1 reference frames, and AMPD with $\mathrm{N}=3$.

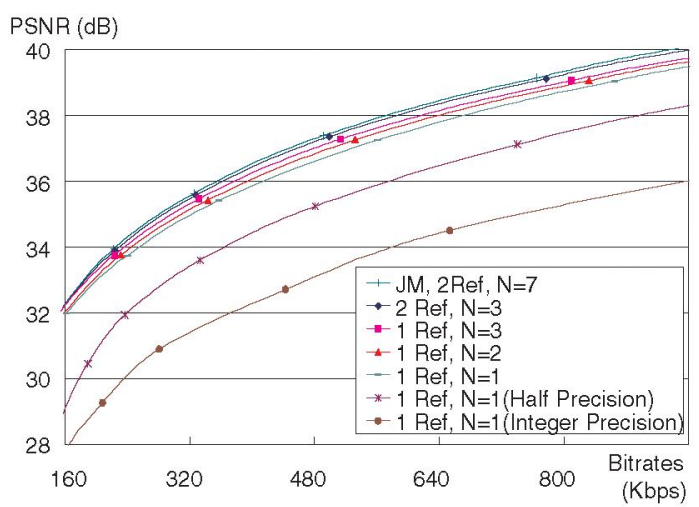

Fig. 7. The rate-distortion curves of the proposed hardware with power aware functionality. The test video is foreman, CIF and $30 \mathrm{frames} / \mathrm{s}$.

the same MV. Therefore, a $4 \times 4$-element PU is designed and reused for all larger blocks by folding technique. For the low power consideration, the intra-candidate and inter-candidate DR schemes are applied. For intra-candidate DR, each $4 \times 4$-element $\mathrm{PU}$ is arranged with four degrees of parallelism to process four horizontally adjacent pixels of one candidate. For horizontal filtering, six integer pixels are required for interpolating one half pixel, while night integer pixels are required for four half pixels. Most horizontally adjacent integer pixels can be reused by horizontal filters, and the on-chip memory BW of SW SRAMs can be reduced. For inter-candidate DR, we arrange nine $4 \times 4$ element PUs to process the nine candidates simultaneously. In this way, the interpolated fractional pixels can be reuse by these $4 \times 4$-element PUs. The redundant computation of interpolation can be saved. Besides, the on-chip memory BW of SW SRAMs can be further reduced. Table I summarizes the performance of candidate level DR for the proposed parallel architecture. After candidate-level DR, the reference pixel data can be reused 20-times more efficiently.

\section{IMPLEMENTATION AND Simulation RESUlT}

The proposed low-power architecture with power-aware fast algorithm is implemented in TSMC $0.18 \mu 1 \mathrm{P} 6 \mathrm{M}$ technology. The total logic gate count is $125 \mathrm{~K}$ with maximum operation frequency of $27 \mathrm{MHz}$. This design can support real-time encoding CIF 30fps videos with two MRF. The supported SR is \pm 32 horizontally and \pm 16 vertically. Figure 6 shows the power reduction performance of logic circuits (SRAM excluded) after the low-power techniques that are applied on each level. Note that Synopsys Prime Power is used to estimate the power consumption here. The innovations on algorithm and architecture levels contribute the most power reduction. Totally $87 \%$ of power con-

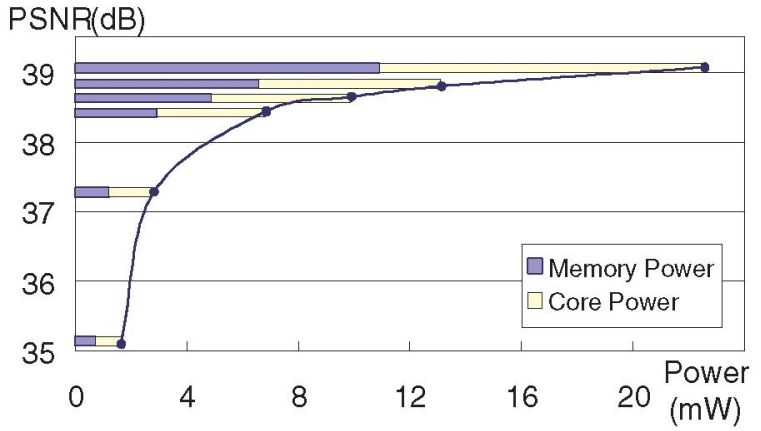

Fig. 8. Performance of power aware functionality. The parameters are foreman $\mathrm{CIF}$ video, 30frames/s, and 700kbps.

sumption in FME is saved after the optimization in each level.

Figure 7 shows the compression performance of our design. The best rate-distortion curve has only $0.1 \mathrm{~dB}$ quality drop compared with reference software. Because the tasks of FME can be dynamically turned off according to the content information, our design can gracefully vary the compression performance in response to the power status. The power consumption versus compression performance is shown in Fig. 8. The power of SW SRAMs is included. Our design can gracefully vary the compression performance of $0.1-3.9 \mathrm{~dB}$ with the $22.58-1.64 \mathrm{~mW}$ power consumption, and is very efficient for different mobile applications in variable power situations.

\section{CONCLUSION}

In this paper, we contributed a low-power and power-aware design for FME of H.264/AVC. The fast algorithm of AMPD is proposed to not only reduce computation complexity, but also provide efficient adaptivity between quality and power. The efficient memory hierarchy and reconfigurable SW SRAMs are used to achieve MB-level data reuse, while the parallel architecture is designed to achieve candidate-level data reuse. As shown in experimental results, the $87 \%$ of power consumption can be saved after low power considerations. The power consumption of $22.58-1.64 \mathrm{~mW}$ can be efficiently varied at expense of $0.1-$ 3.9 dB quality drop.

\section{REFERENCES}

[1] Joint Video Team, Draft ITU-T Recommendation and Final Draft International Standard of Joint Video Specification, ITU-T Recommendation H.264 and ISO/IEC 14496-10 AVC, May 2003.

[2] T. Wiegand, G. J. Sullivan, G. Bjøntegaard, and A. Luthra, "Overview of the H.264/AVC video coding standard," IEEE Transactions on CSVT, 2003.

[3] M. Miyama, J. Miyakoshi, Y. Kuroda, K. Imamura, H. Hashimoto, and M. Yoshimoto, "A sub-mW MPEG-4 motion estimation processor core for mobile video application," IEEE Joumal of Solid-State Circuits, 2004.

[4] S.-S. Lin; P.-C. Tseng; C.-P. Lin; L.-G. Chen;, "Multi-mode content-aware motion estimation algorithm for power-aware video coding systems," in Proceedings of IEEE Workshop on SIPS, 2004.

[5] H.-W. Cheng and L.-R. Dung, "A content-based methodology for poweraware motion estimation architecture," IEEE Transactions on CASII, 2005.

[6] T.-C Chen, Y.-W. Huang, and L.-G. Chen, "Fully utilized and reusable architecture for fractional motion estimation of H.264/AVC," in Proceedings of ICASSP, 2004.

[7] Joint Video Team Reference Software JM8.5, http://bs.hhi.de/ suehring/tml/download/, Sept. 2004.

[8] Y.-W. Huang, B.-Y. Hsieh, T.-C. Wang, S.-Y. Chien, S.-Y. Ma, C.-F. Shen, and L.-G. Chen, "Analysis and reduction of reference frames for motion estimation in MPEG-4 AVC/JVT/H.264," in Proceedings of ICASSP, 2003.

[9] J. C. Tuan, T. S. Chang, and C. W. Jen, "On the data reuse and memory bandwidth analysis for full-search block-matching VLSI architecture," IEEE Transactions on CSVT, 2002. 